\title{
Semiclassical impulse approximation for L-shell electron capture in asymmetric heavy-ion collisions
}

\author{
D H Jakubassa-Amundsen \\ Physik-Department, Technische Universität München, Garching, West Germany
}

Received 13 January 1981, in final form 23 March 1981

\begin{abstract}
The impact parameter dependence of the capture of a target L-subshell electron by a light projectile is calculated within the semiclassical impulse approximation (SCIA). The anisotropy of the resulting target $2 p$ vacancies is strongly dependent on impact parameter and projectile energy. As an example, $(\mathrm{p}, \mathrm{Ar})$ and $(\mathrm{p}, \mathrm{Ne})$ collisions are studied and the capture cross sections are compared with experimental data.
\end{abstract}

\section{Introduction}

Inner-shell electron capture by fast projectiles is a field of current interest in atomic physics. The study of charge transfer has been extended recently to cases with a large asymmetry between the nuclear charges $Z_{1}$ and $Z_{2}$ of the collision partners. For very high collision energies, the total capture cross section is dominated by the capture from the target $\mathrm{K}$-shell, and a considerable number of experiments have been performed on total cross sections as well as on impact parameter $(b)$ distributions (Macdonald et al 1974, Cocke et al 1976, Rødbro et al 1979, Horsdal Pedersen et al 1979). For collision velocities $v$ below the electronic orbiting velocity of the target $\mathrm{K}$ shell, capture from higher shells becomes increasingly important. The first measurements were carried out on the L-shell capture cross section (Rødbro et al 1979, Horsdal Pedersen and Loftager 1981), as well as to some extent on the L- and M-subshell capture cross sections, by studying the resulting charge state distribution of the target atom (Horsdal Pedersen and Larsen 1979).

Theoretical investigations have mostly been limited to the transfer of $\mathrm{K}$-shell electrons in light targets (Mapleton 1972). The transfer of electrons from higher shells of heavy targets has only been calculated within the Brinkman-Kramers theory (e.g. Nikolaev 1967), and recently also using a two-state approach (Lin and Tunnell 1979), but the validity of these theories is questionable especially at high collision energies. There also exists a second-order Born calculation for capture from higher shells, but only in the limit of very fast collisions (Lapicki and Losonsky 1977).

A theory which should be valid not only for fast collisions, but also for slow, asymmetric ones, is the impulse approximation (McDowell and Coleman 1970), formulated in its semiclassical version by Briggs (1977). It is a first-order theory in the (weak) projectile field, while the (strong) target field is included to all orders by introducing a set of intermediate target continuum eigenstates $\psi_{k}^{\mathrm{T}}$, such that charge transfer can be described as ionisation to a high-lying state with a subsequent capture by the projectile. Stimulated by the success of this theory for capture from the K shell 
(Jakubassa-Amundsen and Amundsen 1980) it is extended in this paper to the capture of L-shell electrons. Section 2 contains a derivation of the capture probabilities, and in $\S 3$ total cross sections and zero impact-parameter capture probabilities are evaluated in the case of proton-argon and proton-neon collisions, respectively, and compared with experiment. The impact parameter distribution of the subshell capture probabilities is calculated in $\S 4$, and the anisotropy of the resulting target vacancies is deduced. Concluding remarks follow ( $(5)$. Atomic units $(\hbar=m=e=1)$ are used throughout this paper unless otherwise indicated.

\section{SCIA for capture from the $L$ shell}

In the semiclassical impulse approximation the transfer amplitude from an initial target state $\psi_{i}^{\mathrm{T}}$ to a projectile bound state $\psi_{f}^{\mathrm{P}}$ is given by

$$
a_{f i}=-\mathrm{i} \int_{-\infty}^{\infty} \mathrm{d} t \int \mathrm{d} \boldsymbol{k}\left\langle\psi_{f}^{\mathrm{P}} \mid \boldsymbol{k}\right\rangle\left\langle\psi_{\boldsymbol{k}}^{\mathrm{T}}\left|V_{\mathrm{P}}\right| \psi_{i}^{\mathrm{T}}\right\rangle
$$

where $V_{\mathrm{P}}$ is the projectile field and $|\boldsymbol{k}\rangle$ is a plane wave with momentum $\boldsymbol{k}$. In this notation, the wavefunctions are time dependent and refer to the target rest frame. When the internuclear motion is described by a straight-line path, $\boldsymbol{R}=\boldsymbol{b}+\boldsymbol{v} t,(2.1)$ can be cast into the form (Jakubassa-Amundsen and Amundsen 1980)

$$
\begin{aligned}
a_{f i}=\mathrm{i} \frac{Z_{1}}{\pi} \int \mathrm{d} \boldsymbol{q}_{0} & \exp \left(i \boldsymbol{q}_{0} \cdot \boldsymbol{b}\right) \delta\left(\Delta E-\frac{1}{2} v^{2}+\boldsymbol{q}_{0} \cdot \boldsymbol{v}\right) \\
& \left.\times \int \frac{\mathrm{d} s}{s^{2}} \boldsymbol{\varphi}_{f}^{* \mathrm{P}}\left(\boldsymbol{q}_{0}+\boldsymbol{s}-\boldsymbol{v}\right)<\psi_{q_{0}+\boldsymbol{s}}^{\mathrm{T}}(\boldsymbol{r})|\exp (\mathrm{is} \cdot \boldsymbol{r})| \psi_{i}^{\mathrm{T}}(\boldsymbol{r})\right\rangle .
\end{aligned}
$$

There, use has been made of the Fourier representation of $V_{\mathrm{P}}$ and of the change of variables $\boldsymbol{q}_{0}=\boldsymbol{k}-\boldsymbol{s .} \varphi_{f}^{\mathrm{P}}$ is the Fourier transform of the final state (in the projectile rest frame) and $\Delta E=E_{f}^{\mathrm{P}}-E_{i}^{\mathrm{T}}$ is the energy difference between final and initial electronic state. The ionisation matrix element in (2.2) can be calculated analytically for any initial state if hydrogenic wavefunctions are used, as it can be expressed in terms of derivatives of the integral (McDowell and Coleman 1970, p 364)

$$
I=\int \mathrm{d} \boldsymbol{r} \exp (-\mathrm{i} \boldsymbol{k} \cdot \boldsymbol{r})_{1} F_{1}(\mathrm{i} \eta, 1, \mathrm{i}(k r+\boldsymbol{k} \cdot \boldsymbol{r})) \exp (\mathrm{is} \cdot \boldsymbol{r}) \exp (-Z \boldsymbol{r})
$$

with respect to $Z$ or (and) the transferred momentum $\boldsymbol{s}$ (for fixed final momentum $\boldsymbol{k}$ ). ${ }_{1} F_{1}$ is a confluent hypergeometric function and $\eta=Z_{2} / k$; if $Z=Z_{2}, I$ is proportional to the $1 \mathrm{~s}$ ionisation matrix element. Therefore it is convenient to introduce spherical components $s_{+}, s_{-}$and $s_{z}$ (with $s_{ \pm}=2^{-1 / 2} s \sin \vartheta_{s, v} \exp \left( \pm \mathrm{i} \varphi_{s}\right)$ ) where the $z$ direction is chosen along $v$, such that

$$
\boldsymbol{s} \cdot \boldsymbol{r}=s_{+} r_{-}+s_{-} r_{+}+s_{z} z \text {. }
$$

For a $2 \mathrm{p}, m$ initial state, for example, one needs the derivatives $\mathrm{d} I / \mathrm{d} s_{z}($ for $m=0)$ and $\mathrm{d} I / \mathrm{d} s_{\mp}$ (for $m= \pm 1$ ). The matrix element follows as

$$
\begin{aligned}
& \left\langle\psi_{\mathbf{q}_{0}+s}^{\mathrm{T}}|\exp (\mathrm{is} \cdot \boldsymbol{r})| \psi_{2 \mathrm{p}, m}^{\mathrm{T}}\right\rangle \\
& \quad=c_{1} \exp (\pi \eta / 2) \Gamma(1-\mathrm{i} \eta) \frac{\left[s^{2}-\left(k+\mathrm{i} Z_{2} / 2\right)^{2}\right]^{-\mathrm{i} \eta-1}}{\left(\boldsymbol{Z}_{2}^{2} / 4+q_{0}^{2}\right)^{2-\mathrm{i} \eta}}\left(M_{1} q_{0 \lambda}-M_{2} s_{\lambda}\right)
\end{aligned}
$$




$$
\begin{aligned}
& c_{1}=\mathrm{i} Z_{2}^{7 / 2}\left(m^{2}+m-1\right) /(2 \pi) \quad k=\left(q_{0}^{2}+s^{2}+2 q_{0} s \cos \vartheta_{s, q_{0}}\right)^{1 / 2} \\
& M_{1}=(1-\mathrm{i} \eta)\left((2+\mathrm{i} \eta)+(2-\mathrm{i} \eta) \frac{s^{2}-\left(k+\mathrm{i} Z_{2} / 2\right)^{2}}{Z_{2}^{2} / 4+q_{0}^{2}}\right) \\
& M_{2}=\mathrm{i} \eta(1-\mathrm{i} \eta)+(1+\mathrm{i} \eta)(2+\mathrm{i} \eta) \frac{Z_{2}^{2} / 4+q_{0}^{2}}{s^{2}-\left(k+\mathrm{i} Z_{2} / 2\right)^{2}}
\end{aligned}
$$

with $\lambda=z$ for $m=0$ and \pm for $m= \pm 1$, and $\Gamma$ denoting the gamma function.

It is further possible, as for a $1 \mathrm{~s}$ initial state, to evaluate the intergrals over the angles $\varphi_{q_{0}}$ and $\varphi_{\mathrm{s}}$ in (2.2) analytically (as we consider a $1 \mathrm{~s}$ final state). For the $2 \mathrm{~s}$ initial state, no additional dependence on these angles is introduced by the ionisation matrix element. For the $2 p, m=0$ state, the additional dependence on $\varphi_{s}$ enters through

$$
s_{z}=s\left(\cos \vartheta_{s, q_{0}} \cos \vartheta_{q_{0}}+\sin \vartheta_{s, q_{0}} \sin \vartheta_{q_{0}} \cos \varphi_{s}\right)
$$

and the integration over $\varphi_{s}$ can easily be done. In order to extract the $\varphi$ dependence of the matrix element (2.5) in the case of a $2 \mathrm{p}, m= \pm 1$ state, we write

$$
M_{1} q_{0 \pm}-M_{2} s_{ \pm}=2^{-1 / 2} \exp \left( \pm \mathrm{i} \varphi_{q_{0}}\right)\left\{M_{1} q_{0} \sin \vartheta_{q_{0}}-M_{2} s \sin \vartheta_{s, v} \exp \left[ \pm \mathrm{i}\left(\varphi_{s}-\varphi_{q_{0}}\right)\right]\right\}
$$

and express $\cos \left(\varphi_{s}-\varphi_{q_{0}}\right)$ by means of the addition theorem:

$$
\cos \left(\varphi_{s}-\varphi_{q_{0}}\right)=\frac{\cos \vartheta_{s, q_{0}}-\cos \vartheta_{q_{0}} \cos \vartheta_{s, v}}{\sin \vartheta_{q_{0}} \sin \vartheta_{s, v}}
$$

Inserting this into the last term of (2.6) gives the simple rational expression $\sin \vartheta_{s, v} \exp \left[ \pm \mathrm{i}\left(\varphi_{s}-\varphi_{q_{0}}\right)\right]$

$$
=\cos \vartheta_{s, q_{0}} \sin \vartheta_{q_{0}}-\cos \vartheta_{q_{0}} \sin \vartheta_{s, q_{0}} \cos \varphi_{s} \pm \mathrm{i} \sin \vartheta_{s, q_{0}} \sin \varphi_{s}
$$

which allows an analytical evaluation of the $\varphi$ integrals. While the contribution of the last term in (2.8) vanishes when integrated over $\varphi_{s}$, which leads to the same absolute value of the transition amplitude for $m=+1$ and -1 , the contribution of the second term in $(2.8)$ is proportional to

$$
\begin{aligned}
& \int_{0}^{2 \pi} \mathrm{d} \varphi_{s} \cos \varphi_{s} \varphi_{f}^{* \mathrm{P}}\left(q_{0}+s-v\right)=-\left(2 Z_{1}\right)^{5 / 2} \frac{\beta}{\left(\alpha^{2}-\beta^{2}\right)^{3 / 2}} \\
& \alpha=Z_{1}^{2}+q_{0}^{2}+v^{2}+s^{2}-2 q_{0} v \cos \vartheta_{q_{0}}+2 q_{0} s x-2 v s \cos \vartheta_{q_{0}} x \\
& \beta=-2 v s \sin \vartheta_{q_{0}}\left(1-x^{2}\right)^{1 / 2}
\end{aligned}
$$

with $x=\cos \vartheta_{s, q_{0}}$. The integral over $\varphi_{q_{0}}$ yields the Bessel function $J_{1}$ due to the factor $\exp \left( \pm \mathrm{i} \varphi_{q_{0}}\right)$ in $(2.6)$. Thus the transition amplitude follows as

$$
\begin{aligned}
& a_{f i}(m=0)=\frac{2^{5 / 2}}{\pi v}\left(Z_{1} Z_{2}\right)^{7 / 2} \int_{q_{\min }}^{\infty} \mathrm{d} q_{0} q_{0} J_{0}\left(q_{0} b \sin \vartheta_{q_{0}}\right) \int_{0}^{\infty} \mathrm{d} s \\
& \quad \times \int_{-1}^{1} \mathrm{~d} x M_{0}\left\{M_{1} \alpha q_{0} \cos \vartheta_{q_{0}}-M_{2} s\left[\alpha x \cos \vartheta_{q_{0}}-\beta\left(1-x^{2}\right)^{1 / 2} \sin \vartheta_{q_{0}}\right]\right\} \\
& a_{f i}(m=1)=- \mathrm{i} \frac{4}{\pi v}\left(Z_{1} Z_{2}\right)^{7 / 2} \int_{q_{\min }}^{\infty} \mathrm{d} q_{0} q_{0} J_{1}\left(q_{0} b \sin \vartheta_{q_{0}}\right) \int_{0}^{\infty} \mathrm{d} s \\
& \times \int_{-1}^{1} \mathrm{~d} x M_{0}\left\{M_{1} \alpha q_{0} \sin \vartheta_{q_{0}}-M_{2} s\left[\alpha x \sin \vartheta_{q_{0}}+\beta\left(1-x^{2}\right)^{1 / 2} \cos \vartheta_{q_{0}}\right]\right\}
\end{aligned}
$$




$$
M_{0}=\exp (\pi \eta / 2) \Gamma(1-\mathrm{i} \eta) \frac{\left[s^{2}-\left(k+\mathrm{i} Z_{2} / 2\right)^{2}\right]^{-\mathrm{i} \eta-1}}{\left(Z_{2}^{2} / 4+q_{0}^{2}\right)^{2-\mathrm{i} \eta}}\left(\alpha^{2}-\beta^{2}\right)^{-3 / 2}
$$

where $q_{\min }=q_{0}\left|\cos \vartheta_{q_{0}}\right|=|-\Delta E / v+v / 2|$. For the $2 s$ state, one finds

$$
\begin{aligned}
a_{f i}(2 s)=\mathrm{i} \frac{2^{5 / 2}}{\pi v} & Z_{1}^{7 / 2} Z_{2}^{5 / 2} \int_{q_{\min }}^{\infty} \mathrm{d} q_{0} q_{0} J_{0}\left(q_{0} b \sin \vartheta_{q_{0}}\right) \int_{0}^{\infty} \mathrm{d} s \int_{-1}^{1} \mathrm{~d} x \\
& \times M_{0} \alpha\left[(1+\mathrm{i} \eta)\left(2\left(Z_{2}^{2} / 4+q_{0}^{2}\right)+\frac{1}{2} \mathrm{i} Z_{2} k(2+\mathrm{i} \eta)^{2} \frac{Z_{2}^{2} / 4+q_{0}^{2}}{s^{2}-\left(k+\mathrm{i} Z_{2} / 2\right)^{2}}\right)\right. \\
& +(1-\mathrm{i} \eta)\left(2\left(s^{2}-\left(k+\mathrm{i} Z_{2} / 2\right)^{2}\right)-Z_{2}^{2}(2+\mathrm{i} \eta)\right. \\
& \left.\left.-\frac{1}{2} Z_{2}^{2}(2-\mathrm{i} \eta) \frac{s^{2}-\left(k+\mathrm{i} Z_{2} / 2\right)^{2}}{Z_{2}^{2} / 4+q_{0}^{2}}\right)\right] .
\end{aligned}
$$

The three integrals have to be evaluated numerically. It is straightforward to show that for an arbitrary initial state the transition amplitude can be reduced to an expression of the type (2.10), which is, however, rather lengthy for the higher shells. To prove this, one can make use of the recurrence relations of the Legendre polynomials and follow the lines of the appendix of Jakubassa-Amundsen and Amundsen (1980).

From (2.10), the total cross section

$$
\sigma=2 \pi N_{i} \int_{0}^{\infty} b \mathrm{~d} b\left|a_{f i}\right|^{2}
$$

can easily be calculated by using the orthogonality of the Bessel functions

$$
\int_{0}^{\infty} b \mathrm{~d} b J_{\lambda}\left(q_{0} b \sin \vartheta_{q_{0}}\right) J_{\lambda}\left(q_{0}^{\prime} b \sin \vartheta_{q_{0}}^{\prime}\right)=\frac{1}{q_{0}} \delta\left(q_{0}-q_{0}^{\prime}\right)
$$

$N_{i}$ is the number of initial subshell electrons. As the transition probabilities are very small, the summation over the initial electronic states is not in conflict with the Pauli principle for the capture into the projectile $\mathrm{K}$ shell.

\section{Numerical results and comparison with the BK theory and experiment}

We have evaluated the capture cross section from the $L$ subshells into the $K$ shell by means of (2.11) with (2.10). Hydrogenic wavefunctions with Slater screening $\left(Z_{2}-\right.$ $4.15)$ and experimental binding energies were used. Figure 1 shows the cross section for the capture of Ar $2 \mathrm{p}$ electrons by protons as a function of collision energy. In the SCIA theory, the cross section has a maximum at an energy which is roughly determined by the resonance condition $\Delta E=\frac{1}{2} v^{2}$ where the lower integration limit $q_{\text {min }}$ in $(2.10 a)$ becomes zero. This follows from the behaviour of the ionisation matrix element (2.5) which is a strongly decreasing function of $q_{0}$ such that the transition amplitude becomes smaller when $q_{\min }$ increases. Thereby one should recall that from a physical point of view the transition probability is largest when the momentum transferred to the electron, $q_{\mathrm{EC}}=\left(E_{f}^{\mathrm{P}}+\frac{1}{2} v^{2}-E_{i}^{\mathrm{T}}\right) / v$, has its minimum value which is also found at $\Delta E=\frac{1}{2} v^{2}$. As in the case of capture from the $\mathrm{K}$ shell, it is $q_{\mathrm{EC}}$ that determines the shape of the transition probability, leading to a smooth dependence on energy. 


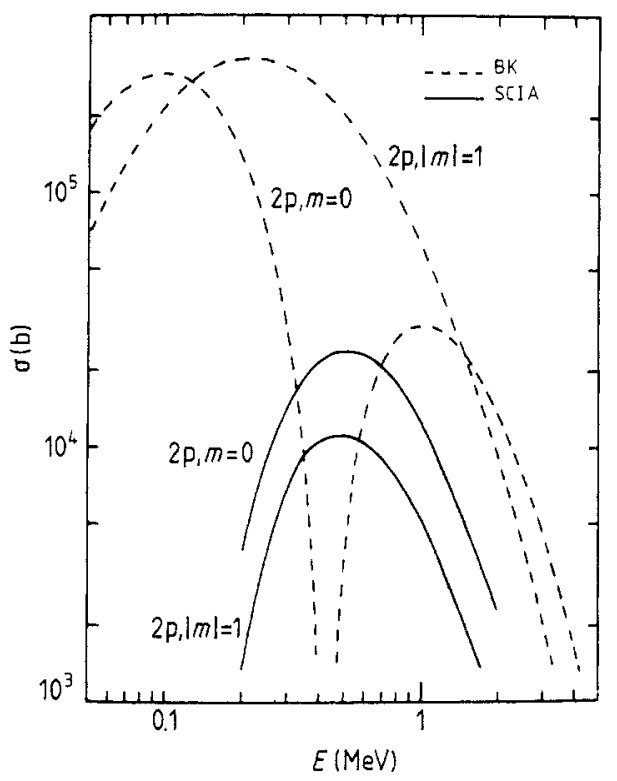

Figure 1. Cross section for electron capture from the 2p subshells of Ar in collisions with protons as a function of projectile energy $E$. The full curves denote SCIA calculations, the broken curves are Brinkman-Kramers results.

Figure 1 also shows the results of the Brinkman-Kramers (BK) theory which are obtained when $\psi_{q_{0}+s}^{\mathrm{T}}$ in (2.2) is replaced by a plane wave. The first-order theory greatly overestimates the cross section for low collision energies. Moreover, in the case of a $2 \mathrm{p}, m=0$ initial state, the $\mathrm{BK}$ cross section has a zero at the resonance energy $(E=0.43 \mathrm{MeV})$. This is due to the fact that the ionisation matrix element becomes independent of the momentum. $s$ in the $\mathrm{BK}$ approximation, and is just given by the Fourier transform $\varphi_{i}^{\mathrm{T}}\left(\boldsymbol{q}_{0}\right)$ of the target wavefunction which (for $2 \mathrm{p}, m=0$ ) is proportional to the vanishing $z$ component of momentum, $q_{o z}=-\Delta E / v+\frac{1}{2} v$. The (unphysical) zero thus results from the lack of an additional coupling (as included in a higher-order theory) to a collision partner with which the momentum can be shared. The difference in the energy distribution of the subshell capture cross sections between the BK and the SCIA approximation indicates that the empirical BK scaling factor $\frac{1}{3}$ which is derived from the high-energy cross section ratio between the second Born approximation (which agrees for very high energies with the impulse approximation) and the BK theory (Lapicki and Losonsky 1977) no longer gives a correct description of the higher-order effects at lower energies. As far as the total L capture cross section is concerned, it is overestimated in the BK theory by a factor much greater than three in the resonance region. On the other hand, a comparison with the few existing L-shell data (figures 2-4) cannot discriminate between the two theories.

Figure 2 shows the L-subshell capture cross sections and a comparison of the total cross sections for $\mathrm{K}$ and $\mathrm{L}$ capture with experiment for ( $\mathrm{p}, \mathrm{Ar}$ ) collisions. In the case of $\mathrm{L}$ capture there are much larger discrepancies between the SCIA and the experimental data than for $\mathrm{K}$ capture. The deviations can only partly be explained by capture into higher projectile states (which are included in the data). A calculation of the capture from the $2 \mathrm{p}, m=0$ state into the $2 \mathrm{p}, m=0$ projectile state along the lines of the 


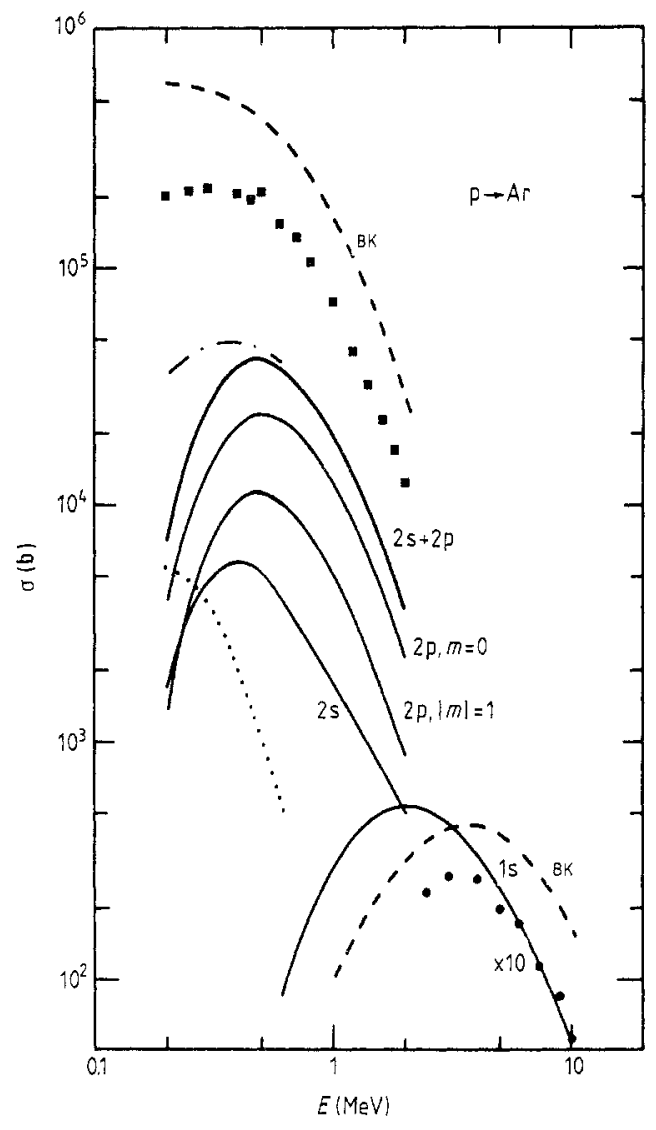

Figure 2. Cross section for electron capture from the $\mathrm{K}$ and $\mathrm{L}$ shell of Ar in collisions with protons as a function of projectile energy $E$. The full curves denote the capture from the L subshells into the $1 \mathrm{~s}$ state, as well as the $K$ and the total $L$ capture cross section into the $1 \mathrm{~s}$ state. The dotted curve is the capture from the $2 \mathrm{p}, m=0$ into the $2 \mathrm{p}, m=0$ state, and the chain curve is the total $\mathrm{L}$ capture including the contribution from excited final states. The broken curves are the Brinkman-Kramers results for capture from the $\mathrm{K}$ and $\mathrm{L}$ shell. The experimental data are from Rødbro et al $(1979 ; \mathbf{\square})$ and from Macdonald et al (1974; $)$.

preceding section shows that at lower energies the capture into excited states can no longer be neglected (figure 2). This indicates the importance of wavefunction-matching effects between initial and final states in slow collisions. As it is very time consuming to include all excited states in the calculation, we estimated their contribution by assuming the cross sections for capture into the three L subshells to be roughly equal, as well as an unchanged relative importance of the initial subshells. This procedure leads to the chain curve in figure 2, producing a shift of the maximum to lower energies, in better agreement with experiment. Another source of discrepancy between theory and experiment is probably the use of hydrogenic wavefunctions in the calculations. It has been shown in the case of L-shell ionisation of $\mathrm{Ar}$ by protons that more accurate wavefunctions may drastically change the ionisation probability (Aashamar and Amundsen 1981), and this correction is much larger than for K-shell ionisation. As the ionisation matrix element enters into the SCIA theory of charge exchange, similar effects are also expected there. On the other hand, there may be an uncertainty in the experimental results as they neglect the difference in the angular distribution of Auger electrons resulting from the decay of the target L-shell vacancies either produced in a capture process or in a direct ionisation process ( $\mathrm{R} \varnothing \mathrm{dbro}$ et al 1979).

In figure 3 the subshell capture probabilities (into the $1 \mathrm{~s}$ state) at zero impact parameter are given in the case of $(\mathrm{p}, \mathrm{Ne}$ ) collisions, and the total capture probability from $\mathrm{Ne}$ is compared with recently performed experiments by Horsdal Pedersen and 


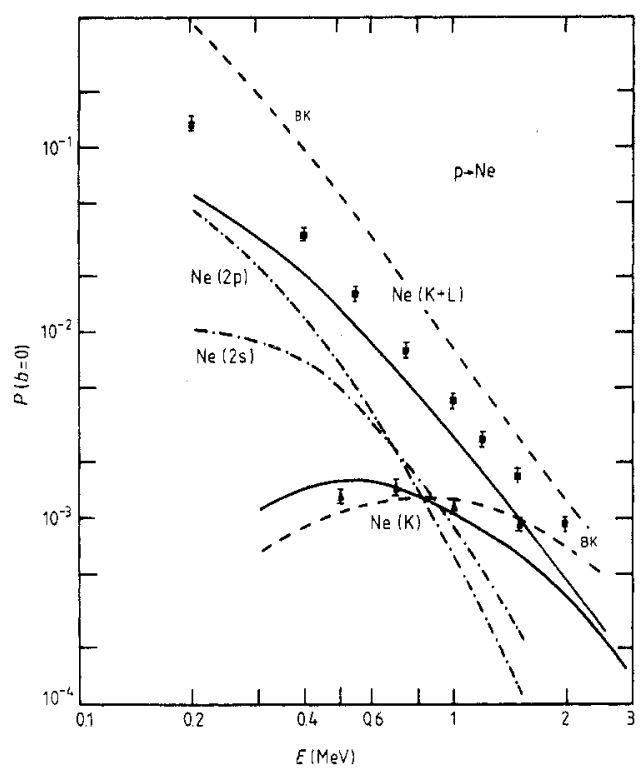

Figure 3. Capture probability at zero impact parameter from the $\mathrm{K}$ and $\mathrm{L}$ shell of $\mathrm{Ne}$ in collisions with protons as a function of projectile energy $E$. The chain curves denote the capture from the $2 \mathrm{p}$ and $2 \mathrm{~s}$ subshells, and the full curves are the capture probability (into the $1 \mathrm{~s}$ state) from the $\mathrm{K}$ shell as well as the sum from $\mathrm{K}$ and $\mathrm{L}$ shell. The broken curves are the corresponding Brinkman-Kramers results. Experimental data for the $\mathrm{K}$ shell ( $\mathbf{C})$ and for total capture ( $)$ are from Horsdal Pedersen and Loftager (1981).

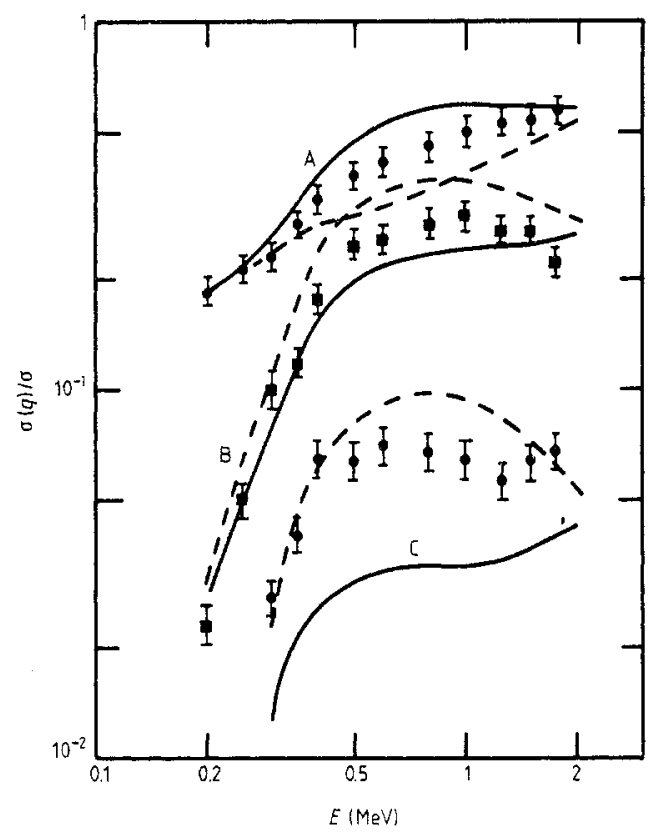

Figure 4. Energy dependence of charge state distributions of $\mathrm{Ar}$ ions resulting from electron capture by protons (A, $q=2 ; \mathrm{B}, q=3 ; \mathrm{C}, q=4$ ). The full curves denote SCIA calculations, the broken curves are the Brinkman-Kramers results. The experimental data are from Horsdal Pedersen and Larsen (1979).

Loftager (1981). At the lower collision energies, the main contribution comes from the $2 \mathrm{~s}$ and the $2 \mathrm{p}, m=0$ initial states (the $2 \mathrm{p}, m=1$ contribution is zero at $b=0$ as it contains the Bessel function $J_{1}$ ), while for energies $E \geqslant 1 \mathrm{MeV}$ the capture from the $\mathrm{K}$ shell becomes important because only the $1 \mathrm{~s}$ state provides the high components of the momentum distribution that are needed for the transition. In the $\mathrm{Ne}$ case, the ScIA reproduces the $\mathrm{L}$-shell capture data better than for Ar. This is probably due to the fact that the ratio of the electronic L-shell orbiting velocity to $v$ is much smaller than for Ar, which reduces the influence of wavefunction effects.

Figure 4 shows the charge state distributions of Ar ions formed in electron capture by protons, i.e. the cross section for detecting an $\mathrm{Ar}$ ion with atomic charge $q$ normalised to the total capture cross section. It can be calculated from the ratio of the $2 \mathrm{~s}$ and $2 \mathrm{p}$ subshell capture cross sections, using the fact that the probability, $N_{q}$, of detecting the charge state $q$ if one $\mathrm{L}$ or $\mathrm{M}$ vacancy is created during the collision, is known experimentally (Horsdal Pedersen and Larsen 1979), and the probability for $\mathrm{M}$-shell capture can also be taken from experiment ( $\mathrm{K}$-shell capture is negligible, see figure 2). The probability for observing charge $q$ is

$$
\sigma(q) / \sigma=N_{q}(2 \mathrm{~s}) \sigma_{2 \mathrm{~s}} / \sigma+N_{q}(2 \mathrm{p}) \sigma_{2 \mathrm{p}} / \sigma+N_{q}(M) \sigma_{\mathrm{M}} / \sigma
$$


with $\sigma_{2 \mathrm{p}} / \sigma=\left(1-\sigma_{\mathrm{M}} / \sigma\right) /\left(\sigma_{2 \mathrm{~s}} / \sigma_{2 \mathrm{p}}+1\right)$ and $\sigma_{2 \mathrm{~s}} / \sigma=\left(\sigma_{2 \mathrm{~s}} / \sigma_{2 \mathrm{p}}\right) \sigma_{2 \mathrm{p}} / \sigma$ where we have used the fact that the sum of the normalised capture cross sections from $L$ and $M$ shells equals one, and $\sigma=\Sigma_{q} \sigma(q)$. There is good agreement with experiment for the cases $q=2$ and 3 , while for $q=4$ only the relative dependence on energy can be reproduced, indicating that the ratio $\sigma_{2 \mathrm{~s}} / \sigma_{2 \mathrm{p}}$ is somewhat too low. Again, the agreement may be improved by using more accurate wavefunctions, as the influence of the other target electrons on the 2 p state is much stronger than on the 2 s state.

\section{Impact parameter dependence and anisotropy}

More detailed tests of collision theories can be obtained by studying the impact parameter distribution of the transfer probability, as well as the anisotropy of the resulting target subshell vacancies. This anisotropy can be expressed by means of alignment and orientation parameters which on one hand are related to the subshell transition amplitudes and cross sections (Fano and Macek 1973) and on the other hand are directly accessible to experiment as they determine the polarisation of light emitted during the subsequent decay of the target vacancies, as well as the anisotropy and spin polarisation of Auger electrons which are an alternative decay mode (Cleff and Mehlhorn 1974). The alignment parameter $A_{0}^{\text {col }}$ is given by

$$
\begin{aligned}
A_{0}^{\mathrm{col}}=\sum_{m}\left[3 m^{2}-l(l+1)\right] P_{m}\left(l(l+1) \sum_{m} P_{m}\right)^{-1} \\
=\frac{\frac{1}{2} P_{|m|=1}-P_{m=0}}{P_{m=0}+P_{|m|=1}} \quad \text { for } l=1
\end{aligned}
$$

where $P_{m}=N_{i}\left|a_{f i}\right|^{2}$ is the subshell capture probability. For an equal population of the magnetic sublevels after the charge transfer, $A_{0}^{\text {col }}$ vanishes which would lead to an isotropic emission of Auger electrons.

In our test case p-Ar we found a great difference in the L-subshell transition probabilities, and thus a large alignment. Figure 5 shows the impact parameter dependence of the capture probabilities, and in figure 6 the alignment as a function of

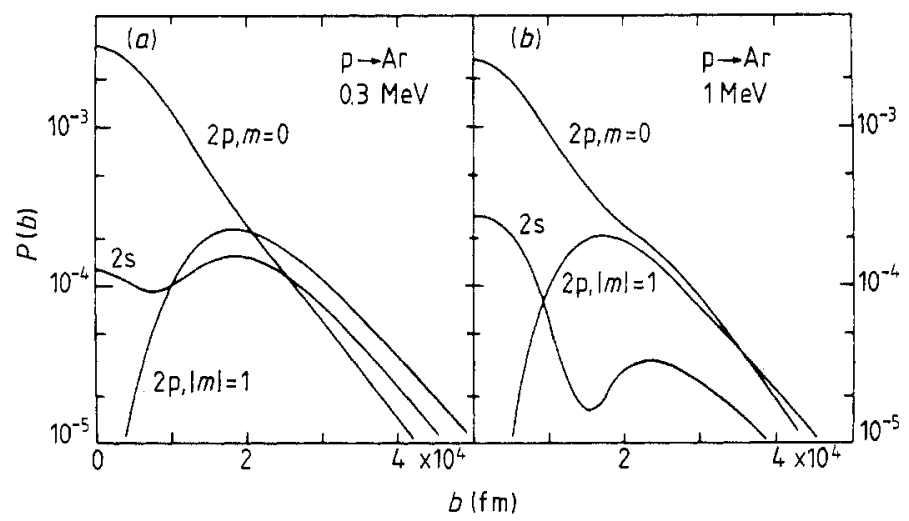

Figure 5. Transition probability for electron capture from the $\mathrm{L}$ subshells of Ar in collisions with protons as a function of impact parameter at collision energies of $(a) 0.3 \mathrm{MeV}$ and $(b)$ $1 \mathrm{MeV}$. 

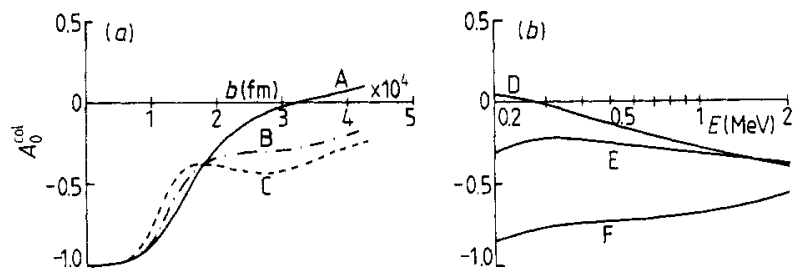

Figure 6. Alignment parameter $A_{0}^{\text {col }}$ characterising the anisotropy of the Ar $2 \mathrm{p}$ subshell vacancies resulting from electron capture by protons, $(a)$ as a function of impact parameter for collision energies: A, $0.3 \mathrm{MeV} ; \mathrm{B}, 1 \mathrm{MeV} ; \mathrm{C}, 2 \mathrm{MeV}$. (b) for fixed impact parameter: D, $3.06 ; \mathrm{E}, 2.14 ; \mathrm{F}, 1.22$ (in units $10^{4} \mathrm{fm}$ ) as a function of $E$.

impact parameter as well as collision energy is given. These calculations are performed for the case of a final 1 s state, but for capture into the $2 \mathrm{p}, m=0$ state we found a very similar $b$ dependence (for a $2 \mathrm{p}, m=0$ initial state) indicating that there may be very little change in anisotropy if the excited projectile states are included. The nonmonotonous impact parameter dependence of the $2 \mathrm{~s}$ capture probability is correlated to the node in the $2 \mathrm{~s}$ wavefunction, and the second maximum in the $b$ distribution becomes very pronounced when the momentum transfer $q_{\mathrm{EC}}$ is close to its minimum value $(E=0.56 \mathrm{MeV})$. At lower or higher energies the structure disappears, which is related to the fact that $q_{\mathrm{EC}}^{-1}$ should be larger than the node radius $2 / Z_{2}$ to make its influence visible. For the $2 \mathrm{p}$ case, the impact parameter dependence can be explained qualitatively by recalling that the $2 p$ wavefunction in coordinate space as well as in momentum space extends parallel to the beam axis (for $m=0$ ) or perpendicular to it (for $|m|=1$ ). For small $b$, one thus has mainly capture from the $m=0$ state (yielding a large, negative alignment). For larger $b$ the $|m|=1$ contribution becomes equally important ( $A_{0}^{\text {col }}$ increases), as the overlap between the projectile state and the target $|m|=1$ state increases (to decrease again for large $b$ ). To account for the energy dependence of $A_{0}^{\text {col }}$, one should note that for small energies the momentum matching is important, leading to the behaviour indicated above, while for high collision energies larger momenta can be transferred, which means an increased probability for capture from $|m|=1$ states for small $b$ and from $m=0$ states for large $b$.

\section{Conclusion}

We have calculated the charge transfer probability from the target $L$ subshells in the semiclassical impulse approximation, but without any further approximations. While for collision velocities near or above the electronic $L$-shell orbiting velocity the capture into the projectile ground state dominates (for a light-heavy collision system), capture into the projectile $\mathrm{L}$ shell becomes important at lower velocities. A comparison of the total cross section for the (p, Ar) system as well as the zero impact parameter capture probability in ( $\mathrm{p}, \mathrm{Ne}$ ) collisions with experimental data shows good agreement in the energy dependence although the absolute values are somewhat too low. This may be due to the insufficiency of hydrogenic wavefunctions for the higher shells which are much more affected by deviations from the Coulomb field than the $\mathrm{K}$ shell. In the impact parameter dependence of the capture probabilities we found large differences between the various subshells, which will result in an anisotropic emission of Auger electrons in the subsequent decay of the target vacancies. Experimental data on the 
impact parameter distribution of the subshell transfer probabilities or on the anisotropy of Auger electrons would be highly desirable to obtain a final answer concerning the applicability of the SCIA for L-shell capture in asymmetric systems.

\section{Acknowledgments}

I should like to thank E Horsdal Pedersen for stimulating this work and for valuable information. I should also like to thank $P$ A Amundsen for discussions and the University of Oslo for the use of their computer facilities.

\section{References}

Aashamar K and Amundsen P A 1981 J. Phys. B: At. Mol. Phys. 14483

Briggs J S 1977 J. Phys. B; At. Mol. Phys. 103075

Cleff B and Mehlhorn W 1974 J. Phys. B: At. Mol. Phys. 7593

Cocke C L, Macdonald J R, Curnutte B, Varghese S L and Randall P 1976 Phys. Rev. Lett. 36782

Fano U and Macek J H 1973 Rev. Mod. Phys. 45553

Horsdal Pedersen E, Folkmann F and Pedersen N H 1979 preprint

Horsdal Pedersen E and Larsen L 1979 J. Phys. B: At. Mol. Phys. 124085

Horsdal Pedersen E and Loftager $P 1981$ private communication

Jakubassa-Amundsen D H and Amundsen P A 1980 Z. Phys. A 297203

Lapicki G and Losonsky W 1977 Phys. Rev. A 15896

L in C D and Tunnell L N 1979 J. Phys. B: At. Mol. Phys. 12 L485

Macdonald J R, Cocke C L and Eidson W W 1974 Phys. Rev. Lett. 32648

McDowell M R C and Coleman J P 1970 Introduction to the Theory of Ion-Atom Collisions (Amsterdam: North-Holland) ch 6-8

Mapleton R A 1972 The Theory of Charge Exchange (New York: Wiley-Interscience)

Nikolaev V S 1967 Sov. Phys.-JETP 24847

Rødbro M, Horsdal Pedersen E, Cocke C L and Macdonald J R 1979 Phys. Rev. A 191936 